\title{
A multi-criteria decision support for optimal instrumentation in scoliosis spine surgery
}

\author{
Marcelo Elias de Oliveira • Carol-Claudius Hasler • \\ Guoyan Zheng • Daniel Studer • \\ Jacques Schneider • Philippe Büchler
}

Received: 16 May 2011 / Revised: 13 October 2011 / Accepted: 14 October 2011 / Published online: 29 November 2011

(C) Springer-Verlag 2011

\begin{abstract}
In adolescent idiopathic scoliosis, the selection of an optimal instrumentation configuration for correcting a specific spinal deformity is a challenging combinatorial problem. Current methods mostly rely on surgeons' expertise, which has been shown to lead to different treatment strategies for the same patients. In this work, a mathematical model of the human spine derived from invitro experimentally-obtained data was used to simulate the biomechanical behavior of the spine under the application of corrective forces and torques. The corrective forces and torques were optimized based on the particle swarm optimization algorithm for each combinatorially possible instrumentation strategy. Finally, a multi-criteria decision support for optimal instrumentation in scoliosis spine surgery has been proposed and applied to five patient data sets exhibiting similar spinal deformities according to two commonly used classification systems. Results indicated that the classification of the spinal deformities based on the current standardized clinical classifications systems is not a sufficient condition for recommending selective fusion of spinal motion segments. In addition, the particle swarm optimization algorithm was successfully applied to solve a realistic interdisciplinary clinical problem in a patient-specific fashion. The proposed method enables a better understanding of the biomechanical behavior of
\end{abstract}

\footnotetext{
M. Elias de Oliveira ( $\varangle)$. G. Zheng · P. Büchler Institut for Surgical Technology and Biomechanics, University of Bern, Stauffacherstr. 78, 3014 Bern, Switzerland e-mail: marcelo.eliasdeoliveira@istb.unibe.ch

C.-C. Hasler · D. Studer · J. Schneider

University Childrens Hospital beider Basel, UKBB,

Spitalstrasse 33, 4056 Basel, Switzerland
}

spinal structures and has the potential to become a standard tool in preoperative planning.

Keywords Adolescent idiopathic scoliosis .

Optimal instrumentation configuration - Interdisciplinary clinical problem $\cdot$ Particle swarm optimization algorithm

\section{Introduction}

Adolescent idiopathic scoliosis (AIS) is a complex threedimensional deformity of the trunk, characterized by deviations of the spine in the sagittal and coronal planes as well as by vertebral axial rotation. The AIS is a musculoskeletal disorder, usually accompanied by rib cage deformity. Its prevalence ranges from 2 to $3 \%$, appearing most frequently during the puberty, out of which about $10 \%$ of the patients require surgical intervention.

The objectives of the surgical intervention are to prevent the curve progression as well as to improve the cosmetic appearance, spinal stability and balance (Bridwell 1999). The correction of the scoliotic curve is achieved by a complex orthopedic instrumentation composed of pedicle screws, spinal rods, vertebral hooks, and cross-links. During the surgical intervention, pedicle screws are judiciously placed in several vertebrae of the patient, so that each pedicle screw is inserted through a vertebral pedicle into the vertebral body, as shown in the inset of Fig. 1. In order to correct the spinal deformity, surgical maneuvers for fixation of the spinal rods into the pedicles screws or vertebral hooks are performed using specific surgical instruments. Consequently, a set of corrective forces and torques capable of correcting a specific spinal deformity is generated. 


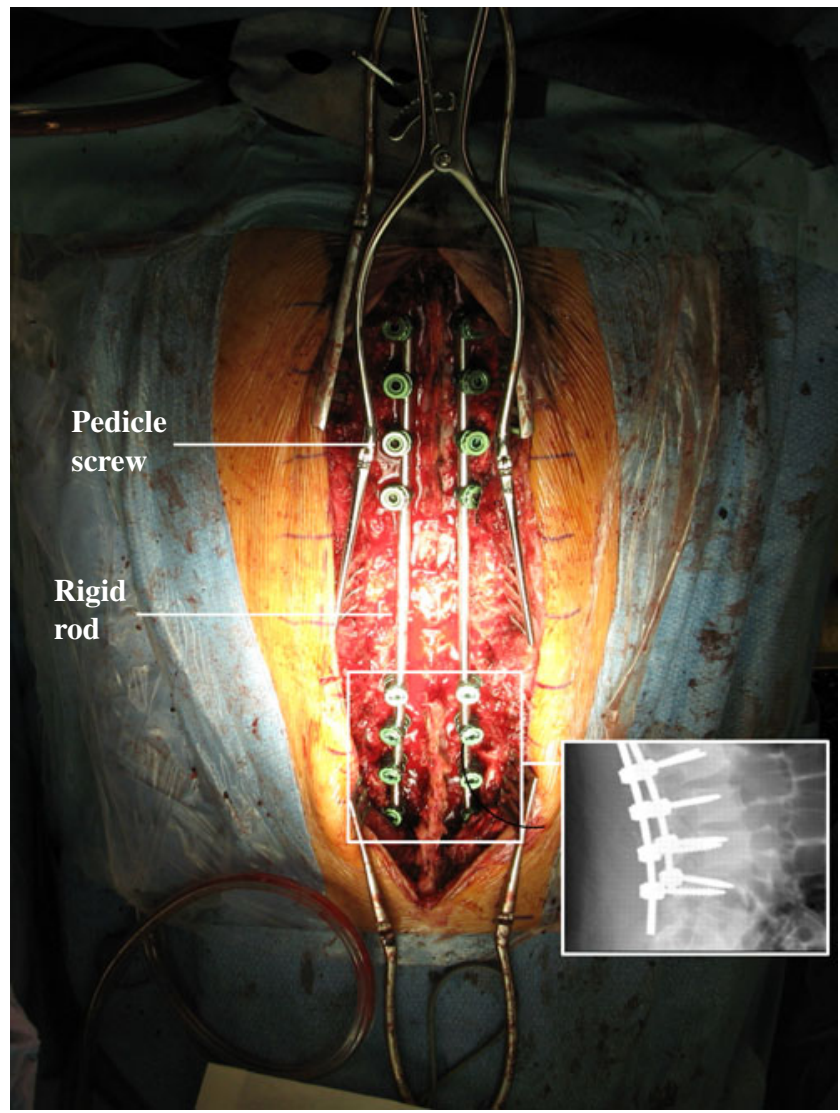

Fig. 1 The surgical procedure is performed with the patient in the prone position. A posterior midline incision is made from the upper end of the spinous process two levels above the uppermost pedicle instrumented to the lowest instrumented vertebra. The spinal deformity is corrected by applying the corrective forces to individual vertebral bodies and connecting the rigid rods to the pedicle screws. The inset shows a postoperative radiograph of the pedicle screws inserted into the vertebral bodies

The selection of fusion levels and the determination of a sequence of surgical maneuvers (i.e., magnitudes and directions of the forces and torques generated by the surgical instruments) are difficult decisions to made by the surgeon, relying mostly on its experience and on its interpretation of subjective and objective clinical informations that are available preoperatively. The objective clinical informations are mostly based on conventional anteroposterior X-ray radiographs, such as the ones shown in Figs. $4 b, 5 b, 6 b, 7 b$, $8 \mathrm{~b}$. During the preoperative planning of spinal surgery, the spinal deformities are clinically classified according to the King and Lenke classifications systems (King et al. 2011; Lenke et al. 2001). These two commonly used clinical classification systems enable surgeons to recommend selective fusion of spinal motion segments, and provide guidelines for evaluating the appropriateness of different surgical interventions. In addition to these techniques, the amount of rotation at the apex of the spine is quantified by using the Cobb angle (Cobb 1948), and its variation during the performance of clinical tests is associated with the spinal flexibility, thus, giving to the surgeon an indication of the amount of correction that could be achieved by the surgical intervention. Since the onset of corrective scoliosis surgery, various clinical tests have been devised to access the spinal flexibility in the coronal plane preoperatively. These include erect or supine side bending radiographs (Cheh et al. 2007; Klepps et al. 2001), fulcrum bending radiographs (Cheung and Luk 2011; Luk et al. 1998), supine traction (Polly and Sturm 1998; Vaughan et al. 1996) and push prone radiographs (Vedantam et al. 2000).

Nonetheless, recent studies have provided substantial evidences that no clear consensus exists among spinal surgeons regarding the identification of ideal surgical interventions for treating spinal deformities. Key questions concerning the selection of fusion levels and appropriate instrumentation systems remain unanswered (Lenke et al. 1998a; Krismer et al. 1992; Puno et al. 2003). Recently, Robitaille et al. (2007) have performed a study involving a significant group of experienced surgeons. In this study, preoperative planning proposed by several surgeons for the same group of patients was characterized by a large variability in the total number of instrumented spinal segments, and by significant variations of the selected inferior and superior instrumented vertebrae. These inconsistent results in determining an optimal instrumentation configuration for correcting a specific spinal deformity can be attributed to subjective criteria such as the surgeon's experience and its interpretation of published clinical practice guidelines.

To overcome these problems, several mathematical models have been created to predict the biomechanical behavior of the human spine. The first numerical models were proposed in the 1990s and were based on nonlinear finite element method (Gardner-Morse and Stokes 1994; Stokes and Gardner-Morse 1999; Leborgne et al. 1999). These models are limited by unknown patient-specific mechanical properties, and most of these models presented several convergence difficulties due to the complexity of the material laws, displacements fields, and boundary conditions (Stokes et al. 1999). Recent works aiming at modeling the behavior of spinal structures are based on differential algebraic equations (DAEs) arising from multibody system dynamics (Poulin et al. 1998; Petit et al. 2004; Aubin et al. 2003) and linear finite element analysis (Ghista et al. 1988; Vanderby et al. 1986; Gardner-Morse and Stokes 1994; Dumas et al. 2005). Most of these models have been characterized based on the pioneer experimentally work of Panjabi et al. (1976), which described an elegant measurement system and reported systematically obtained, threedimensional flexibility and stiffness coefficients of spinal motion segments from healthy fresh human cadaver thoracic 
spines considering coupling effects. Based on this work, Gardner-Morse et al. (1990), presented a method for deriving a spinal element stiffness matrix based on shear beam model, matching numerical simulations to the reported experimental observations. Furthermore, these models have been proved to be a good alternative for describing and predicting the biomechanical behavior of the spinal column in posterior spinal instrumentation. However, the determination of optimal instrumentations strategies were not addressed in these previous studies. Thus, each individual surgical strategy has to be individually simulate, beside being the definition of a surgical instrumentation a complex task and time consuming. Nevertheless, the determination of all possible instrumentation configuration by the user is a prohibitive or impossible task to be achieved, since the number of possible instrumentation strategies increases exponentially with the number of vertebrae in the region of interest.

In this work, the spinal instrumentation configuration was formulated as a combinatorial problem and the finite element approach proposed by Gardner-Morse et al. (1990) was used for modeling of spinal motion segments. The optimal corrective forces and torques were determined based on the particle swarm optimization algorithm for each combinatorially possible instrumentation strategy. Finally, a multi-criteria decision support for optimal instrumentation in scoliosis spine surgery has been proposed to determine a subgroup of optimal surgical instrumentation strategies. In addition, this approach has been applied to five patients exhibiting similar scoliotic deformities according to standardized classifications systems.

\section{Methods}

The methodology proposed in this work to determine optimal surgical instrumentations strategies is summarized in the flow chart shown in Fig. 2.

\subsection{Patient-specific spine deformity}

Five patients with AIS requiring surgical intervention were considered in this study. Preoperative curve patterns were classified according to the Lenke and King classification systems (King et al. 2011; Lenke et al. 2001).

Concave and convex sides of the spinal column were approximated by cubic B-spline via an interactive selection of control points, thus, allowing an automatic computation of the spinal midline. Finally, a semi-automatic method for detection of the intersections between the computed spinal midline with the upper and lower vertebral endplates was used to create patient-specific finite element meshes
Spine geometry and FE mesh generation

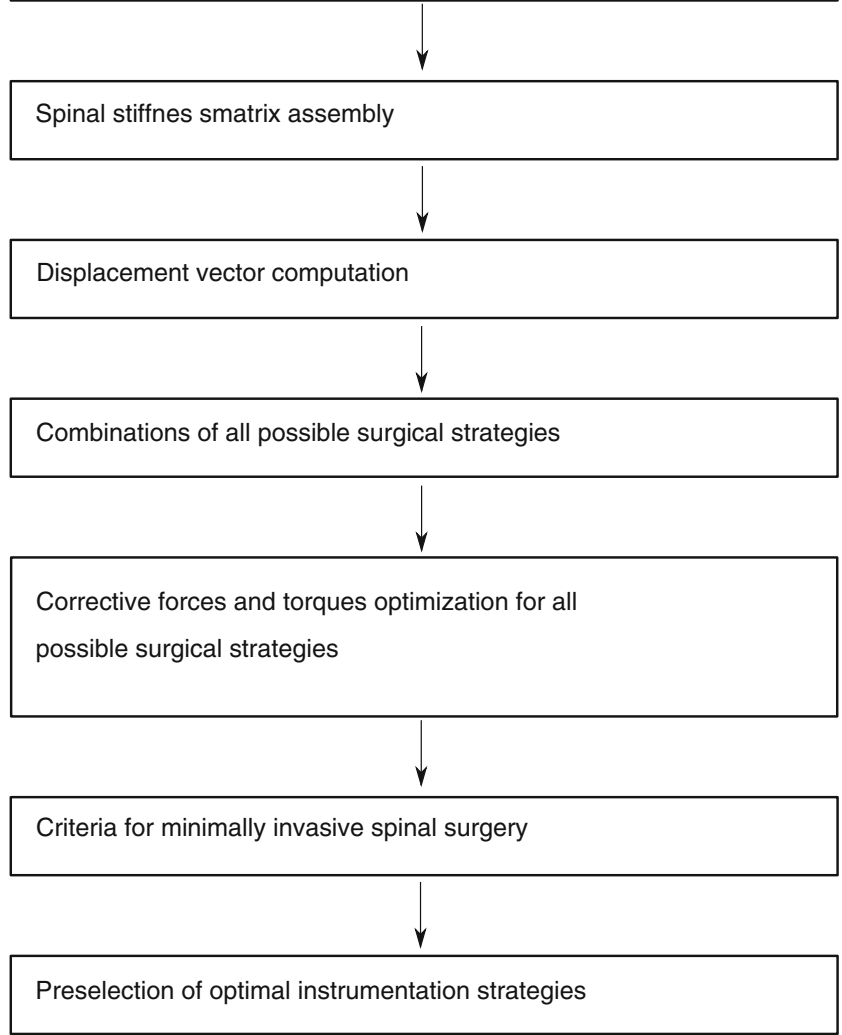

Fig. 2 Flow chart summarizing the proposed methodology for recommending selective fusion of spinal motion segments

(Fig. 2c), as described elsewhere (Elias de Oliveira et al. 2011).

\subsection{Spinal finite element model formulation}

In 1976, Panjabi et al. have shown that the spinal segments are characterized by motion coupling patterns and these relationships were converted into flexibility and stiffness coefficients. In this previous work, the measurements were performed in a right-hand cartesian coordinate system as shown in Fig. 2b, the region of interest was characterized by a displacement vector resulting from six load-independent components, and the center of the upper and lower vertebral bodies were chosen as the origin of these two coordinate systems (Fig. 3b). In the late 1990s, focusing on the understanding of the human spine biomechanical behavior, Gardner-Morse et al. (1990) have derived a spinal element stiffness matrix based on shear beam model, reproducing all the essential features of the previously reported experimental observations performed by Panjabi et al. (1976), representing the three-dimensional flexibility and stiffness properties of the human spine in a spinal stiffness matrix 
(a)

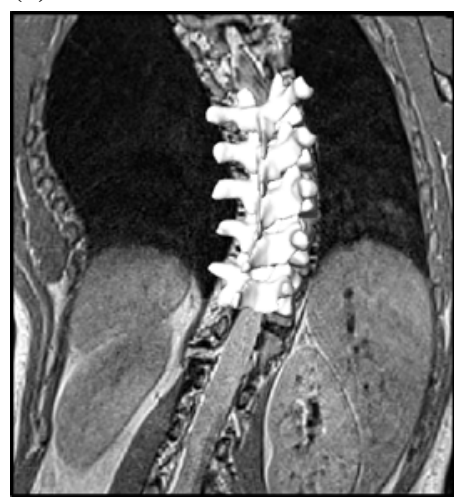

(c)

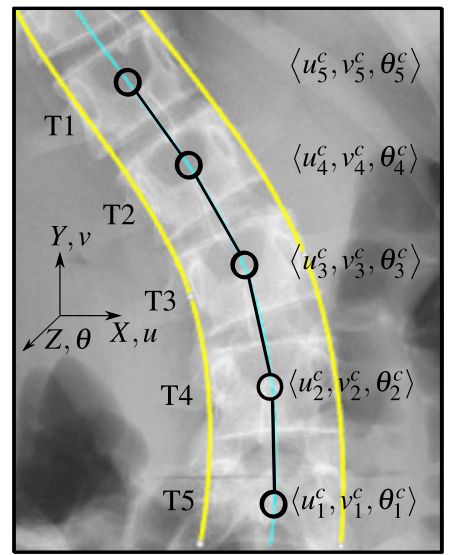

(b)

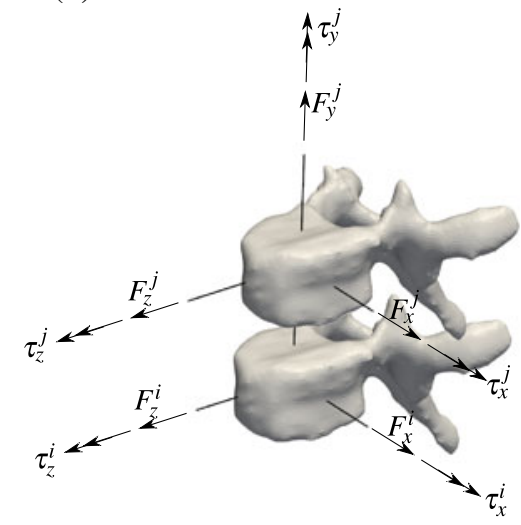

(d)

$$
K_{l}=10^{4} .\left[\begin{array}{cccccc}
0.011 & 0 & 0.164 & -0.011 & 0 & 0.171 \\
& 0.124 & 0 & 0 & -0.124 & 0 \\
& & 19.7 & -0.164 & 0 & -14.71 \\
& \text { Sym. } & & 0.011 & 0 & -0.171 \\
& & & & 0.124 & 0 \\
& & & & & 19.909
\end{array}\right]
$$

Fig. 3 (a) Magnetic resonance image (MRI) of a scoliotic spine. In this volumetric data set, five thoracic vertebrae were segmented to illustrate the existence of vertebral axial rotation along the spine column. (b) Spinal segment motion coordinate system adopted by Panjabi et al. (1976) for the characterization of spine mechanical properties-two-node structure with six degrees of freedom per node.

composed by $12 \times 12$ elements. This matrix is generated from a $6 \times 6$ stiffness matrix relating the displacement vector with six components (three translations and three rotations) to the load vector acting on it, and the distances between two adjacent vertebral bodies centroids were determined by assuming static equilibrium and by minimizing the differences between the apparent stiffness of the two nodes of a specific spinal motion segment.

Since the onset of corrective scoliosis surgery, the preoperative planning for surgical treatment is based on conventional anteroposterior standing radiographs. In the present study, spinal motion segments were modeled as a two dimensional structure, thus, the terms associated with rotations and translations in the sagittal and transverse planes were neglected, as shown in Fig. 3c. Therefore, the spinal element stiffness is reduced to $6 \times 6$ terms, since only three degrees of freedom per node are preserved.

Based on the finite element approximation, the relations between nodal forces and displacements for a given (c) Patient-specific finite element mesh obtained from a conventional anteroposterior X-ray radiograph. The spinal structure is modeled based on a two-node shear beam element with three degrees of freedom per node. (d) Spinal element stiffness matrix derived from in-vitro data measurements in human cadaver thoracic spines (Panjabi et al. 1976; Gardner-Morse et al. 1990)

spinal element motion segment $l$ are represented by the local stiffness equation:

$\left\{F_{l}\right\}_{6 \times 1}=\left[K_{l}\right]_{6 \times 6}\left\{\delta_{l}\right\}_{6 \times 1}$,

where $\left[K_{l}\right]$ is the spinal element stiffness of an element $l$ (Fig. 2d); $\left\{F_{l}\right\}$ and $\left\{\delta_{l}\right\}$ are forces and displacement vectors represented in the element coordinate system, respectively. For a given element $l$ possessing two nodes $i$ and $j$, and with three degrees of freedom per node, the displacement vector is defined by the difference between corrected spine deformity and the current spine geometric form in the element coordinate system, i.e., $\delta_{l}=\left[\left(u_{i}^{c}-u_{i}^{0}\right),\left(v_{i}^{c}-v_{i}^{0}\right)\right.$, $\left.\left(\theta_{i}^{c}-\theta_{i}^{0}\right),\left(u_{j}^{c}-u_{j}^{0}\right),\left(v_{j}^{c}-v_{j}^{0}\right),\left(\theta_{j}^{c}-\theta_{j}^{0}\right)\right]^{T}$, where the superscript index $c$ refers to the theoretically corrected spine; $u_{i}$ and $v_{i}$ are the components of the displacement vector of the node $i$ in the $x$ and $y$ directions, respectively; $\theta_{i}$ is the angle of rotation normal to the mid-surface of the corrected 
spine; and the superscript index 0 refers to the patientspecific spinal geometry before starting the optimization procedure.

It is important to note that during the surgical intervention, the forces and torques are mainly transmitted by means of the pedicle screws which are fixed into the vertebral bodies, as shown in the inset of Fig. 1. Therefore, the corrective forces and torques are applied to the nodes of the finite element mesh in order to simulate the surgical maneuvers, considering the fact that a two-node shear beam element is defined between two consecutive vertebral bodies with nodes located in their respective centroids.

The local spinal element stiffness were merged into the global stiffness matrix by appropriate indexing and matrix-entry addition as described elsewhere (Bathe 1996; Zienkiewicz et al. 2005). The dimension of the global stiffness matrix is determined by the number of vertebral bodies in the region of interest multiplied by the number of degrees of freedom per node. In our simulations, the spinal finite element structure was assumed to be hinged on the sacrum and the spinal structure was initially rotated so that the most superior vertebral body was aligned with the neutral axis of the theoretically correct spine.

\subsection{Combinatorial analysis for determining the set of corrective forces and torques}

Let the region of interest be composed by $N_{\mathrm{vb}}$ vertebral bodies. Considering the number of instrumented vertebral bodies $\left(N_{\mathrm{ivb}}\right)$ as ranging from one to the total number of vertebral motion segments $\left(N_{\mathrm{vb}}\right)$, the number of distinguishable instrumentation strategies is given by

$2^{N_{\mathrm{vb}}}-1=C_{1,\left(N_{\mathrm{vb}}-1\right)}^{N_{\mathrm{vb}}}+C_{2,\left(N_{\mathrm{vb}}-2\right)}^{N_{\mathrm{vb}}}+\cdots+C_{N_{\mathrm{vb}, 0}}^{N_{\mathrm{vb}}}$, where:

$C_{\mathrm{Nivb},\left(N_{\mathrm{vb}}-N_{\mathrm{ivb}}\right)}^{\mathrm{Nvb}}=\frac{N_{\mathrm{vb}} !}{N_{\mathrm{ivb}} !\left(N_{\mathrm{vb}}-N_{\mathrm{ivb}}\right) !}$,

and $N_{\mathrm{ivb}} \leq N_{\mathrm{vb}}$.

For illustrative purposes, the combinatorial analysis will be performed on a lumbar spine (Fig. 3c) to determine all possible configurations of instrumentations strategies. In this particular case, the region of interest is composed by a set of five vertebral bodies $\left(N_{\mathrm{vb}}=5\right)$. Thus, the amount of combined instrumentation strategies is given by $\left(2^{5}-1\right)$ strategies, where the dimension of the searching space $(\mathcal{D})$ ranges from the number of degrees of freedom per node to the number of vertebrae in the region of interest multiplied by the number of degrees of freedom per node (i.e., 3 to 15). Therefore, the set of all possible surgical instrumentation strategies can be summarized as shown in Table 1 .

\subsection{Cost function}

Formally, let $f: \Re^{\mathcal{D}} \rightarrow \Re$ be defined as a quadratic cost function which must be minimized, where $\mathcal{D}$ is the dimension of the search space, i.e., total number of instrumented vertebral bodies $\left(N_{\mathrm{ivb}}\right)$ multiplied by the number of degrees of freedom per node, which in our case is three. For each patient data set, all possible configurations of instrumentation were calculated by combinatorial analysis according to (2). It is important to note that the dimension of the searching space ranges from three to the total number of instrumented vertebral bodies multiplied by three and that the total number of instrumented vertebral bodies is always less or equal than the number of vertebral bodies $\left(N_{\mathrm{vb}}\right)$ present in the region of interest, i.e., $N_{\mathrm{ivb}} \leq N_{\mathrm{vb}}$.

Table 1 ID: Instrumentation strategy identification number

\begin{tabular}{|c|c|c|c|c|c|}
\hline \multirow{2}{*}{ ID, $\mathrm{N}_{\mathrm{ivb}}, \mathcal{D}$} & \multicolumn{5}{|c|}{ Thoracic vertebrae } \\
\hline & $\mathrm{T} 1$ & $\mathrm{~T} 2$ & $\mathrm{~T} 3$ & $\mathrm{~T} 4$ & T5 \\
\hline $1,1,3$ & $F_{x}^{1}, F_{y}^{1}, \tau_{z}^{1}$ & $0,0,0$ & $0,0,0$ & $0,0,0$ & $0,0,0$ \\
\hline $2,1,3$ & $0,0,0$ & $F_{x}^{2}, F_{y}^{2}, \tau_{z}^{2}$ & $0,0,0$ & $0,0,0$ & $0,0,0$ \\
\hline $3,1,3$ & $0,0,0$ & $0,0,0$ & $F_{x}^{3}, F_{y}^{3}, \tau_{z}^{3}$ & $0,0,0$ & $0,0,0$ \\
\hline . & . & . & . & . & . \\
\hline$\cdot$ & . & . & . & $\cdot$ & . \\
\hline . & . & . & . & . & . \\
\hline $30,4,12$ & $F_{x}^{1}, F_{y}^{1}, \tau_{z}^{1}$ & $F_{x}^{2}, F_{y}^{2}, \tau_{z}^{2}$ & $F_{x}^{3}, F_{y}^{3}, \tau_{z}^{3}$ & $F_{x}^{4}, F_{y}^{4}, \tau_{z}^{4}$ & $0,0,0$ \\
\hline $31,5,15$ & $F_{x}^{1}, F_{y}^{1}, \tau_{z}^{1}$ & $F_{x}^{2}, F_{y}^{2}, \tau_{z}^{2}$ & $F_{x}^{3}, F_{y}^{3}, \tau_{z}^{3}$ & $F_{x}^{4}, F_{y}^{4}, \tau_{z}^{4}$ & $F_{x}^{5}, F_{y}^{5}, \tau_{z}^{5}$ \\
\hline
\end{tabular}

$N_{\text {ivb }}$ : Number of instrumented vertebral bodies. $\mathcal{D}$ : Dimension of the searching space. $F_{x}^{\alpha}, F_{y}^{\alpha}, \tau_{z}^{\alpha}:$ Corrective forces and torques to be optimized based on the particle swarm algorithm, where the superscript index $\alpha$ refers to the spinal motion segment level 
A candidate solution in the form of a vector of dimension $\mathcal{D}$ is taken as argument of the proposed cost function $\mathcal{E}$ and produces a scalar real number as output which indicates the fitness of a given candidate solution. During the optimization procedure, the objective is to find the optimal set of forces and torques $\left\{\mathbf{F}^{*}\right\}$ capable of correcting the spinal deformity by minimizing the proposed cost function (4). In other words, we want to find a set of corrective forces $\mathbf{F}^{*} \subseteq \mathcal{F} \subseteq \mathfrak{R}^{\mathcal{D}}$, such that: $\mathbf{F}^{*}=\underset{\mathbf{F} \in \mathcal{F}}{\operatorname{argmin}} \mathcal{E}(\mathbf{F})=\left\{\mathbf{F}^{*} \in \mathcal{F}: \mathcal{E}\left(\mathbf{F}^{*}\right) \leq \mathcal{E}(\mathbf{F}) \forall \mathbf{F} \in \mathcal{F}\right\}$, where $\mathcal{E}$ is given by

$\mathcal{E}(\mathbf{F})=\left(N_{\mathrm{vb}} \times d o f\right)^{-1}\{\mathbf{e}\}\{\mathbf{e}\}^{T}$,

$\{\mathbf{e}\}=\{\mathbf{u}\}-\{\delta\}$,

$\{\mathbf{u}\}=[\mathbf{K}]^{-1}\{\mathbf{F}\}$,

where $\{\mathbf{u}\}=\left[u_{1}, v_{1}, \theta_{1}, \ldots, u_{\left(N_{\mathrm{vb}} \times 3\right)}, v_{\left(N_{\mathrm{vb}} \times 3\right)}, \theta_{\left(N_{\mathrm{vb}} \times 3\right)}\right]$ is the resulting displacement vector obtained by applying the optimized force vector $\left\{\mathbf{F}^{*}\right\} ;\{\mathbf{e}\}$ is the error vector, arising from the difference between the displacement vector $\{\mathbf{u}\}$ and the theoretically computed displacement vector $\{\delta\} ;[\mathbf{K}]$ is the global stiffness matrix of the spinal structure; $\{\mathbf{F}\}$ is the set of forces and torques for correction of the spine deformity, which is a $\left(N_{\mathrm{vb}} \times d o f\right)$-dimensional parameter vector with $\mathcal{D}$ components to be optimized, where $d o f \leq$ $\mathcal{D} \leq N_{\mathrm{vb}} \times d o f$; and $\mathcal{E}(\mathbf{F})$ is the cost function to be minimized, which is a scalar measure defined as the mean square error between the computed displacement vector $\{\mathbf{u}\}$ and the desired displacement vector $\{\delta\}$ defined in Section 2.2.

It is important to point out, that only one instrumentation configuration among all possible configuration strategies considers all vertebral bodies as being instrumented, i.e.,

$C_{N_{\mathrm{Nvb}}, 0}^{N_{\mathrm{Nv}}}=1$, (3). For all other strategies, the respective components of the vector $\{\mathbf{F}\}$ for non-instrumented vertebral bodies were assumed to be zero, since corrective forces and torques were not applied to all spine segments during the optimization procedure.

\subsection{Particle swarm optimization algorithm}

The particle swarm optimization algorithm (PSOA) was proposed in 1995 by Kennedy and Eberhard and is described in details elsewhere (Kennedy and Eberhart 1995; Shi and Eberhart 1998). This algorithm is a bio-inspired computational model, exploiting cooperative and social behavior's heuristics, such as observed in fish schooling, birds flocking, and insects swarming. The PSOA consists of a population of distributed candidate solutions immersed in a space of arbitrary dimension. During the optimization process, the particles tend to move toward the best solution in the search-space. The dynamics of each individual particle is influenced by individual and social components, i.e., its own previous best experience and the previous global best performance of the swarm.

Considering a swarm composed by $n p$ particles, at time $t>0$, the position of the $i$-th particle $\mathbf{x}_{i}$ is updated by adding its respective velocity vector $\mathbf{v}_{i}$, which is a function of its own experience, i.e., its best previous fitness $\mathbf{p}_{i}$ and of the global best fitness $\mathbf{p}_{g}$ found among all particles of the swarm. The particle's speed is stochastically adjusted according to (7):

$\mathbf{v}_{i}^{t+1}=\omega \mathbf{v}_{i}^{t}+c_{1} r_{1}\left(\mathbf{p}_{i}^{t}-\mathbf{x}_{i}^{t}\right)+c_{2} r_{2}\left(\mathbf{p}_{g}^{t}-\mathbf{x}_{i}^{t}\right)$,

and particle's position is position is update by

$\mathbf{x}_{i}^{t+1}=\mathbf{x}_{i}^{t}+\mathbf{v}_{i}^{t+1}$,

where $\mathbf{x}_{i}$ and $\mathbf{v}_{i} \in \Re^{\mathcal{D}} ; r_{1}$ and $r_{2}$ are random numbers ranging from 0.0 to $1.0 ; c_{1}$ and $c_{2}$ are the learning factors, which were assumed to be equal to 2.0 (Kennedy and Eberhart 1995); and $\omega$ is the particle's moment ranging from 0.9 to 1.2 according to Shi and Eberhart (1998). Lower and upper boundaries of the search-space, $x_{\text {lower }}, x_{\text {upper }}$, respectively, were defined for setting the initial positions of the $i-t h$ particles $\left(\mathbf{x}_{i}^{0}\right)$. The initial domains of the search-space were defined based on the maximal absolute values computed by solving the linear system of equations derived from the finite element formulation.

In evolutionary methods, the population size is an important aspect, reflecting directly in the robustness and computation cost of the algorithm. In this regard, a small population size may converge to a local minimum. In another hand, large population will increase the computational cost and may be characterized by slow convergence rates. Thus, different populations should be tested for verification of stable solutions (i.e, a solution which is invariant to the population size). In our experiments, we range the initial population from 10 to 100 particles, incrementing by 10 the size of the population. It was verified that the optimized forces have not undergone considerable changes, when selecting a population with 20 or more candidate solutions. Thus, an initial population composed by 20 particles was used in our optimization procedure. The used PSOA algorithm for determining the set of forces and torques capable of correcting spinal deformities in a patient-specif fashion is described in pseudo-code Algorithm 1.

2.6 Criteria for minimally invasive spinal surgery and dimensionality reduction

An important point to mention is that during the optimization procedure, the cost function previously defined (4) is 


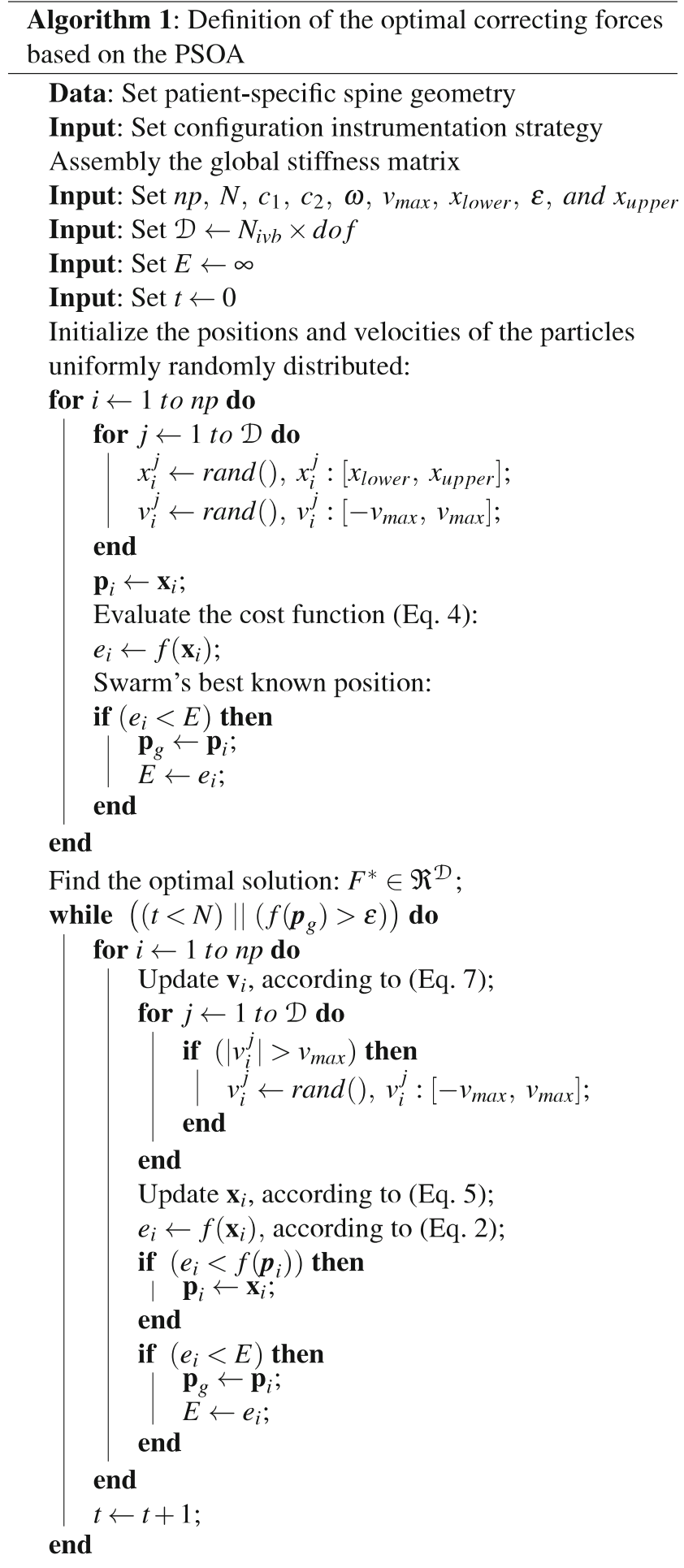

minimized and the stop criterion resulted from the standard formulation takes into account only the geometric aspects of the spine. In this sense, an optimal instrumentation strategy can not be differentiated based solely on the finiteelement element formulation, since the spinal deformity is always corrected by applying the optimized set of corrective forces and torques. However, different forces and torques are needed for correcting a specific spinal deformity using a specific instrumentation configuration. In order to compare the magnitude of the forces and torques, the maximum absolute value for the corrective forces and torques observed among all optimized strategies was selected. Consecutively, forces and torques for all optimized strategies were represented with respect to this value, thus, making it possible to differentiate these optimized instrumentation strategies in terms of forces and torques. In this section, important aspects in spine surgery will be intrinsically represented by three different criteria. According to this formulation, an optimal instrumentation strategy is characterized by an effective correction of the spinal deformity by applying relatively low levels of corrective forces and by considering a small number of instrumented vertebral bodies, thus, being a minimally invasive surgical treatment. These criteria have been used to preselect a subset of best optimal spinal instrumentation strategies, and are defined below.

i) Correctiveness of the spinal deformity: This criterion is computed based on the mean square error between the displacement vector obtained by applying the optimized force vector $\left\{\mathbf{F}^{*}\right\}$ and the theoretically computed displacement vector $\{\delta\}$ (defined in Section 2.2).

ii) Definition of the upper limits of safety: This criterion can be defined only after optimizing all possible instrumentation strategies, since the magnitude of the forces and torques for correcting a specific spinal deformity is expressed relative to the global maximum estimated quantity. This normalization procedure ensures the exchange of information between different surgical procedures, thus, all forces and torques are linearly mapped into the interval $(0,1]$.

iii) Minimal invasiveness: This criterion is defined as being the relation between the total number of instrumented levels with the selected inferior and superior instrumented vertebrae, $V_{\text {sup }}$, and $V_{\text {inf }}$, respectively, given by the following expression: $\left(V_{\text {sup }}-V_{\text {inf }}\right) \leq$ $N_{\text {ivb }}+1$, thus allowing a maximum of two nonconsecutive instrumented vertebral bodies between the selected inferior and superior instrumented vertebrae.

The first and second criteria were normalized for each patient data set between zero and one. A variable called $\lambda$ was introduced for reducing the problem dimensionality, and was defined as the average of the magnitudes of the first and second previously defined criteria, i.e., $\lambda$ : $\mathfrak{R}^{2} \rightarrow \Re \in(0,1]$, thus, the optimized surgical instrumentation strategies can be represented in a two-dimensional space, where the abscissa axis shows the parameter $\lambda$ which 
Table 2 Spine deformity classified according to the Lenke and King classification systems

\begin{tabular}{|c|c|c|c|c|}
\hline \multirow[t]{2}{*}{ Patient } & \multicolumn{2}{|c|}{ Classification } & \multirow[t]{2}{*}{$2^{\mathrm{Nvb}}-1$} & \multirow[t]{2}{*}{$\mathcal{D}$-ranging } \\
\hline & Lenke & King & & \\
\hline Patient 1 & $1 \mathrm{~A}$ & III & $2^{14}-1=16,383$ & $3-42$ \\
\hline Patient 2 & $1 \mathrm{~A}$ & IV & $2^{14}-1=16,383$ & $3-42$ \\
\hline Patient 3 & $1 \mathrm{~A}$ & IV & $2^{13}-1=8,191$ & $3-39$ \\
\hline Patient 4 & $1 \mathrm{C}$ & II & $2^{16}-1=65,535$ & $3-48$ \\
\hline Patient 5 & $1 \mathrm{~A}$ & IV & $2^{13}-1=8,191$ & $3-39$ \\
\hline
\end{tabular}

The total number of possible instrumentation strategies is calculated based on the total number of vertebral bodies included in the finite element model. The dimension of the solution search space ranges from three to the number of instrumented vertebral motion segments multiplied by the number of degrees of freedom per spinal element node, which is three in this two-dimensional study. It is important to note that the total number of possible instrumentation strategies increases exponentially with the number of considered vertebral bodies

is a dimensionless quantity, while the ordinate axis shows the preselected optimal strategies achieved for different amount of instrumented vertebrae, where $1 \leq N_{\text {ivb }} \leq N_{\mathrm{vb}}$. According to our assumption, an optimal instrumentation strategy is a minimally invasive surgery (i.e., small number of instrumented vertebral motion segments) characterized by an effective correction of the spine deformity by applying relatively low levels of corrective forces and torques.

We would like to stress that the terminology optimal instrumentation strategies is intended to add focus to the fact that an optimal instrumentation configuration can be defined for different amount of instrumented vertebral bodies. Let us define a region of interest composed of $N_{\mathrm{vb}}$ vertebrae. Thus, the total number of distinguishable instrumentation strategies is given by the sum of all possible configurations for $N_{\text {ivb }}$ instrumented vertebrae, where $N_{\text {ivb }}$ ranges from 1 to $N_{\mathrm{vb}}$. Considering an instrumentation strategy $\mathbf{S}_{\mathrm{ivb}}$ composed of $N_{\mathrm{ivb}}$ vertebrae, thus, the $\mathbf{S}_{\mathrm{ivb}}$ is a vector with $\frac{N_{\mathrm{vb}} !}{N_{\mathrm{ivb}} !\left(N_{\mathrm{vb}}-N_{\mathrm{ivb}}\right) !}$ components, as defined in Section 2.3. Each component of the vector $\mathbf{S}_{\mathrm{ivb}}$ is characterized by its respective $\lambda$. Therefore, the optimal instrumentation strategy considering $N_{\text {ivb }}$ vertebrae to be instrumented is defined by the $\inf _{\lambda \in \Lambda_{\text {ivb }}}\left\{\mathbf{S}_{\mathrm{ivb}}\right\}$ and the global optimal instrumentation strategy is defined by the $\min \left\{\inf _{\lambda \in \Lambda_{1}}\left\{\mathbf{S}_{1}\right\}, \inf _{\lambda \in \Lambda_{2}}\left\{\mathbf{S}_{2}\right\}, \ldots, \inf _{\lambda \in \Lambda_{\mathrm{ivb}}}\left\{\mathbf{S}_{\mathrm{ivb}}\right\}\right\}$, where $\Lambda_{\alpha}$ is the set of all surgical instrumentation strategies considering $\alpha$ instrumented vertebral bodies.

\section{Results}

For each of the five patients included in this study, all possible configurations of instrumentation strategies were determined by combinatorial analysis, as discussed in Section 2.3. Optimal corrective forces and torques were calculated for each individual instrumentation strategy based on the PSOA (Algorithm 1). Finally, the criteria for minimally invasive spinal surgery was applied for preselecting optimal instrumentation strategies, and then each surgical (a)

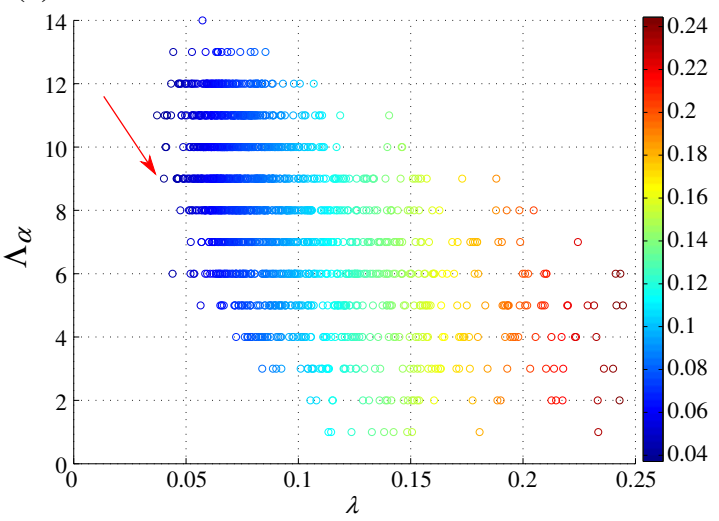

(b)

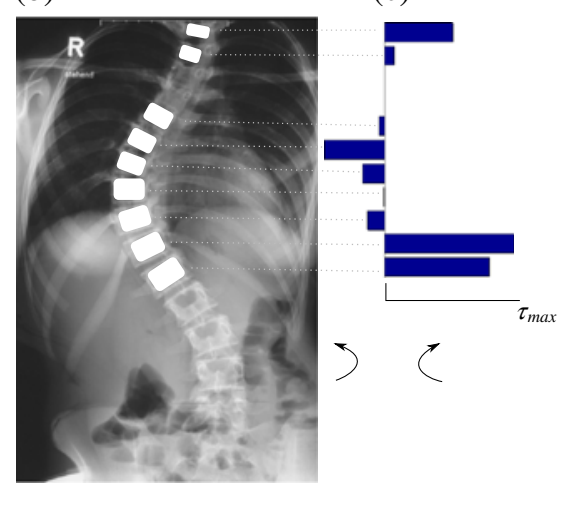

Fig. 4 Patient 1. (a) The abscissa axis shows the parameter $\lambda$ while the ordinate axis shows the preselected optimal instrumentation configuration strategies considering $\alpha$ instrumented vertebral bodies, i.e., $\left\{\forall \lambda \in \Lambda_{\alpha}: \lambda<0.25\right\}$. (b) Preoperative anteroposterior standing radiograph and representation of the selected optimal instrumentation strategy consisting of nine instrumented vertebrae. (c) Optimized torques for correcting the patient-specific spine deformity, where $\tau_{\max }$ represents the maximum torque relative to upper limits of safety 
(a)

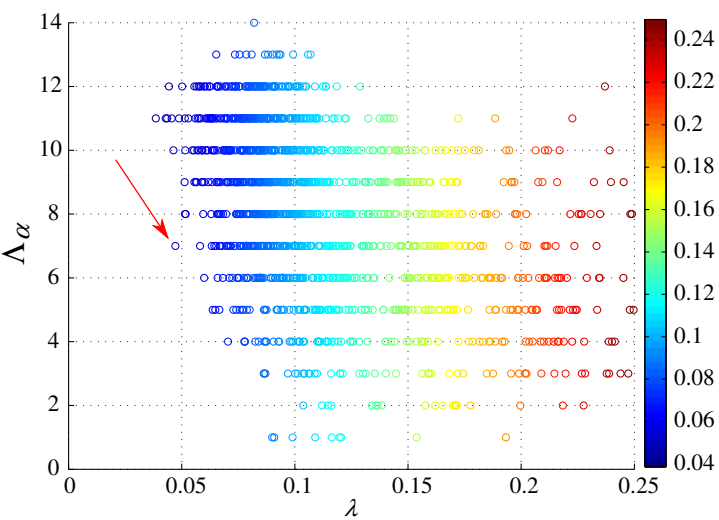

(b)

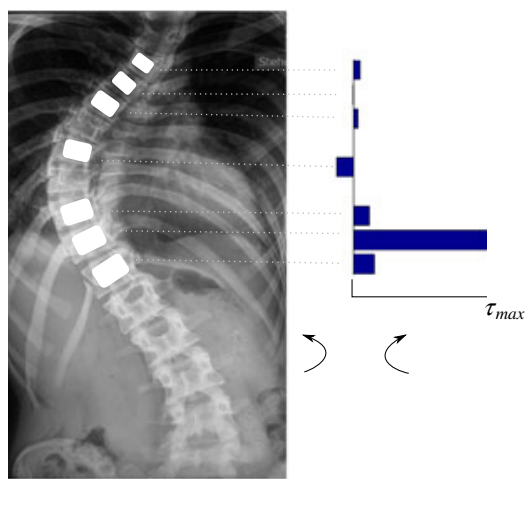

Fig. 5 Patient 2. (a) The abscissa axis shows the parameter $\lambda$ while the ordinate axis shows the preselected optimal instrumentation configuration strategies considering $\alpha$ instrumented vertebral bodies, i.e., $\left\{\forall \lambda \in \Lambda_{\alpha}: \lambda<0.25\right\}$. (b) Preoperative anteroposterior standing radiograph and representation of the selected optimal instrumentation strategy consisting of seven instrumented vertebrae. (c) Optimized torques for correcting the patient-specific spine deformity, where $\tau_{\max }$ represents the maximum torque relative to upper limits of safety (a)

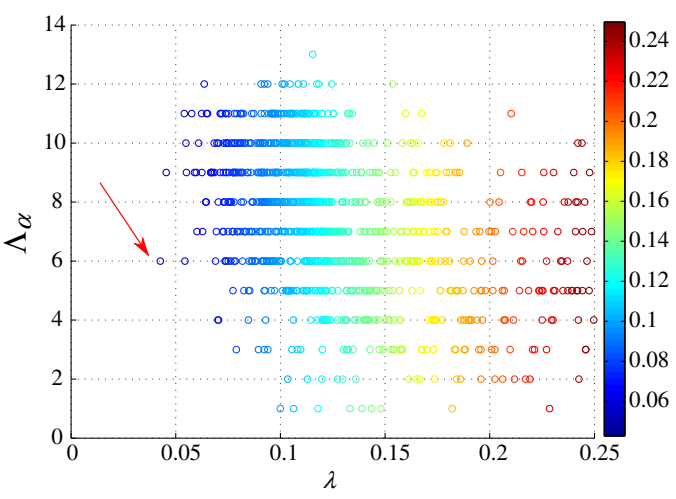

(b)

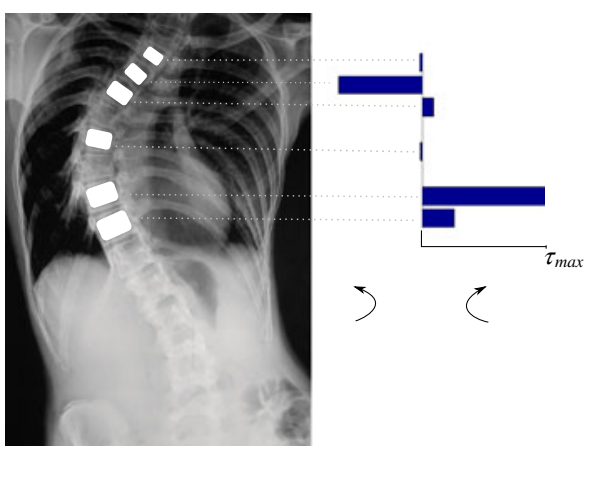

Fig. 6 Patient 3. (a) The abscissa axis shows the parameter $\lambda$ while the ordinate axis shows the preselected optimal instrumentation configuration strategies considering $\alpha$ instrumented vertebral bodies, i.e., $\left\{\forall \lambda \in \Lambda_{\alpha}: \lambda<0.25\right\}$. (b) Preoperative anteroposterior standing radiograph and representation of the selected optimal instrumentation strategy consisting of six instrumented vertebrae. (c) Optimized torques for correcting the patient-specific spine deformity, where $\tau_{\max }$ represents the maximum torque relative to upper limits of safety (a)

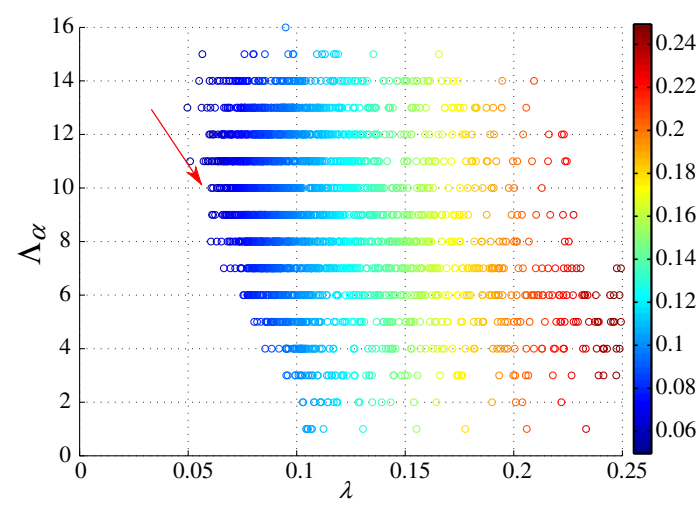

(b)

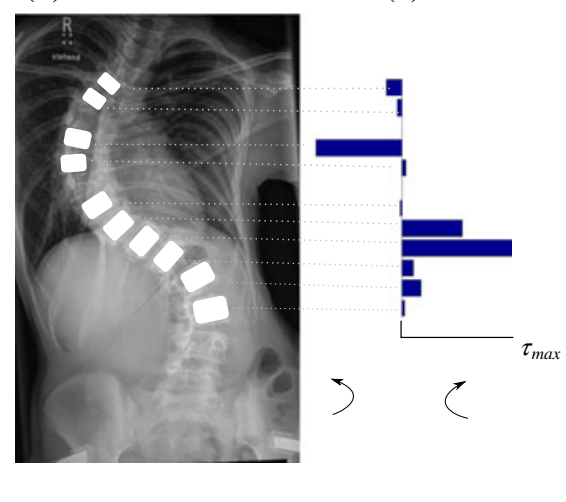

Fig. 7 Patient 4. (a) The abscissa axis shows the parameter $\lambda$ while the ordinate axis shows the preselected optimal instrumentation configuration strategies considering $\alpha$ instrumented vertebral bodies, i.e., $\left\{\forall \lambda \in \Lambda_{\alpha}: \lambda<0.25\right\}$. (b) Preoperative anteroposterior standing radiograph and representation of the selected optimal instrumentation strategy consisting of ten instrumented vertebrae. (c) Optimized torques for correcting the patient-specific spine deformity, where $\tau_{\max }$ represents the maximum torque relative to upper limits of safety 
(a)

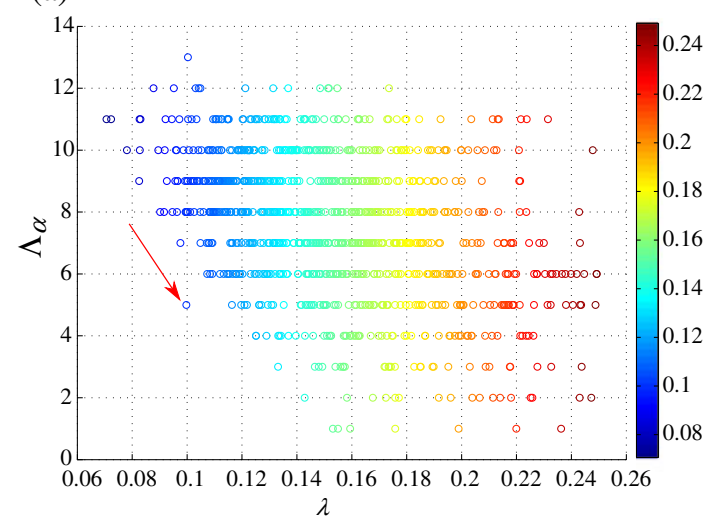

(b)

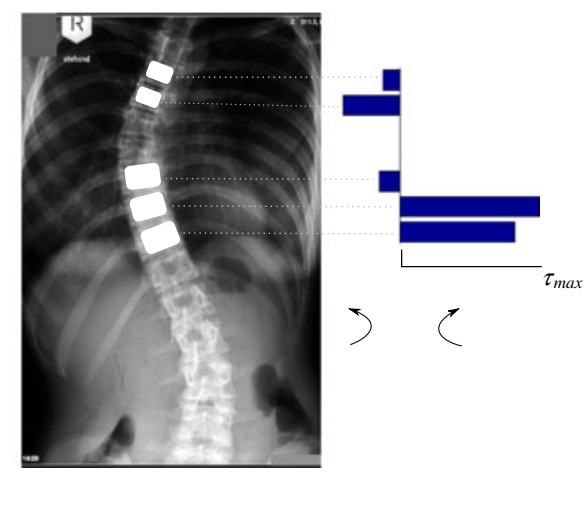

Fig. 8 Patient 5. (a) The abscissa axis shows the parameter $\lambda$ while the ordinate axis shows the preselected optimal instrumentation configuration strategies considering $\alpha$ instrumented vertebral bodies, i.e., $\left\{\forall \lambda \in \Lambda_{\alpha}: \lambda<0.25\right\}$. (b) Preoperative anteroposterior standing

instrumentation strategy was graphically represented by a circle in a two-dimensional space, where the $\lambda$ values were mapped into a color space ranging from blue to red, for low and high values, respectively.

The Table 2 shows the classified spinal deformities according to the Lenke and King classification systems, together with the total number of possible configuration instrumentation strategies, and the search space dimension range. Most of the patients were classified as 1A according to Lenke's classification and as IV according to King's classification.

The outcomes of the proposed multicriteria optimization procedure for all five considered cases were graphically represented (Figs. 4a, 5a, 6a, 7a, 8a), and only optimized instrumentations strategies characterized by a $\lambda<$ 0.25 are considered for illustrative purposes. The proposed multicriteria optimization procedure suggests different combinatorial configurations for spinal instrumentation, considering different number of instrumented vertebral bodies, i.e., ranging from one to the total number of vertebrae in the region of interest $\left(N_{\mathrm{vb}}\right)$. It is important to recall that in our simulations, the discretized spinal structure is assumed to be hinged on the sacrum. Each solution is characterized by a specific set of corrective forces and torques, which are required for correcting a specific spinal deformity, resulting in an approximation of the theoretically computed displacement vector $\{\delta\}$.

For illustrative purposes, a single instrumentation strategy was selected (highlighted by an arrow) and represented in its respective preoperative anteroposterior standing radiograph (Figs. 4b, 5b, 6b, 7b, 8b). The instrumented spinal motion segments were represented by white quadrilaterals, and the optimized torques for correcting the patientspecific spine deformity were graphically displayed in bar-plot form (Figs. 4c, 5c, 6c, 7c, 8c). radiograph and representation of the selected optimal instrumentation strategy consisting of five instrumented vertebrae. (c) Optimized torques for correcting the patient-specific spine deformity, where $\tau_{\max }$ represents the maximum torque relative to upper limits of safety

\section{Discussion}

The selection of optimal surgical configurations strategies is fundamentally a combinatorial problem which requires searching over all possible subsets of surgical strategies. Thus, in this paper we have proposed a novel methodology combining finite-element model of human spinal motion segments with the PSOA to determine optimal spine instrumentation strategies in a patient-specific fashion.

In this work, a two-dimensional finite element model of the human spine was considered for calculating the magnitudes and the directions of the corrective forces. However, the extension of the presented algorithm to three-dimensions is straightforward. Acquisition of threedimensional information on the patient's spine could be achieved using different techniques such as magnetic resonance imaging (MRI), computerized tomography (CT) or three-dimensional stereoradiographic reconstruction from multiple X-ray projections. Furthermore, it is important to note that the proposed method is general, and it can easily be extended to more complex formulations. However, we would like to stress that the derived spinal element stiffness matrix by Gardner-Morse et al. (1990) reproduces all the essential features of the previously reported experimental observations performed by Panjabi et al. (1976), assuming isotropy, and material and geometrical linearity. These assumptions are not valid for large deflections when considering the elementary beam theory. In particular, the elementary beam theory does not take into account the square of the first derivative in the calculation of the curvature of the beam, as well as the correction for the shortening of the moment arm resulted from the deflection of the beam. Therefore, a more robust theory such as the proposed by Bisshopp and Drucker (1945) should be considered when large displacements are involved. 
Clinically, the spinal deformities in AIS are classified according to the King and Lenke classifications systems (King et al. 2011; Lenke et al. 2001). The classification of spinal deformities enables surgeons to recommend specific treatments, and allows intercomparison between different surgical techniques. However, these current classification systems based on standard radiographic measurements, are controversial and characterized by low inter- and intraobserver reliability (Lenke et al. 1998b; Ogon et al. 2002). These classification techniques provide a basic description of changes in spine morphology induced by the pathology, but does not provide a detailed description of the spine geometry, and it fails in providing a characterization of the mechanical properties of the patient's spine, as well as a description of its biomechanical behavior.

The proposed approach suggest a set of instrumentation strategies that ideally correct a specific spinal deformity, but the final decision for selecting a specific instrumentation strategy remains with the surgeon, since the pathology may induce changes in the vertebral bodies, thus, constraining the use of a specific instrumentation and/or the insertion of pedicle screws into determined vertebral pedicles. Additionally, the determination of the surgical procedure must be individualized according to the patient's general medical condition.

Results obtained on the five selected patients clearly show a significant variation in instrumentation strategies for each patient, even for different patients with the same King or Lenke classification. In addition, for each patient, the model proposes different instrumentation configurations with a similar ability to correct a specific spinal deformity. The surgical instrumentation strategies are graphically represented in a two dimensional space, and the assumed relevant surgery-related factors (i.e., correctiveness of the spinal deformity, and the definition of the upper limits of safety) are intrinsically represented by the variable $\lambda$ and are presented in an intuitive manner. In our results, only one instrumentation strategy was represented in its respective preoperative anteroposterior standing radiograph. Nevertheless, the simultaneous visualization of different instrumentation strategies can easily be incorporated into a software-tool for computer-assisted preoperative planning. However, this strategy was not necessarily the global optimal instrumentation strategy, as observed in patient 4 (Fig. 7b). It is important to note that no significant changes have been observed for the optimized instrumentation strategies when considering more than eight instrumented vertebral bodies, as shown in Fig. 7a. Strategies involving a high number of instrumented vertebrae were not represented graphically, illustrating that the correction of a specific spinal deformity can be achieved by considering a lower number of instrumented vertebral bodies, which is an important aspect of this work.
The objectives of the surgical procedure is to prevent curve progression, as well as to improve the cosmetic appearance, spinal stability and postural balance. Intuitively, if we consider a patient with severe scoliosis, e.g., at both lumbar and thoracic region, the geometrical correction of the spinal deformity may be achieved only if a considerable number of vertebrae would be instrumented, which is not the most appropriate situation when considering another aspects for a minimally invasive surgery. Furthermore, this procedure would constrain natural movements and even induce motor control deficits. These points have been addressed in our work and our results showed that geometrical corrections can be achieved with forces and torques of low magnitude, and similar effectiveness of the surgical treatment can be achieved considering different number of instrumented vertebral bodies, thus indicating that the classification of the spinal deformities based on the current standardized clinical classifications systems is not a sufficient condition for recommending selective fusion of spinal motion segments.

The data sets used in the present study correspond to preoperative standing posteroanterior radiographs. One problem with such conventional data acquisition protocols is that the intrinsic parameters of the X-ray imaging system are not estimated, since both a calibration method and a calibration device for stereoradiography are not conventionally used in clinical practice, thus, the projective parameters cannot be estimated. As a consequence, forces and torques cannot be accurately calculated, since measures can only be performed in the image coordinate system. For this reason, only normalized information has been used in the presented optimization procedure and the results should be interpreted relative to upper limits of safety based to the surgeons' experience and published clinical practice guidelines. A compact and low-cost calibration grid could be used in the future during the clinical routine to determine the extrinsic and intrinsic parameters of the X-ray imaging system (Hartley and Zisserman 2000), thus resulting in an accurate determination of the magnitude of the corrective forces and torques. In addition, commonly used surgical instruments are not equipped with force sensors. Therefore, the corrective forces applied during the surgical intervention cannot be quantified precisely, since they are determined by the surgeons' experience. Therefore, the magnitude of the forces and torques were represented symbolically, instead of being representing numerically.

An important aspect of the PSOA is the fact that it is not a gradient based-method, which is especially useful in cases when the gradient cannot be computed efficiently or even impossible to be derived. Non-gradient based methods such as the proposed by Rosenbrock (1960) and by Powell (1964) have been proved to be entirely satisfactory. However, these methods were not tested in our work, but 
they could be employed without requiring any change in the proposed concept. Finally, it is important to note that the surgical instrumentation strategies were considered as a combinatorial optimization, thus, the number of problems to be optimized (i.e., number of possible instrumentation strategies) increases exponentially with the number of considered instrumented vertebral bodies. These computations are performed during the preoperative planning, thus, the computational time could be reduced by considering the parallelization of our algorithm based on PSOA, which has the advantage of being quickly and easily performed when compared to non-evolutionary methods. Nevertheless, it is important to stress that this procedure occurs in the preoperative planning stage (i.e., not in real-time), where time is not necessarily a critical parameter.

Our vision is that in the future, non-invasive techniques for assessment of the spinal mechanical properties will be developed, which will be combined with current imaging techniques for reconstruction of the patient-specific spine geometry, thus, leading to more realistic and accurate biomechanical models of the human spine. The multicriteria decision support for optimal instrumentation proposed in this work has the potential to become a standard technique in the preoperative planning of spinal surgery, since it eliminates the subjectivity in defining optimal instrumentations strategies.

Acknowledgments The authors gratefully acknowledge Prof. Dr. Raphael Haftka (Dept. of Mechanical and Aerospace Engineering., University of Florida, USA) for many helpful comments and suggestions, kindness, and generosity. And a special thanks to the anonymous reviewers who provided thoughtful suggestions, which have enhanced the quality of this work.

\section{References}

Aubin CE, Petit Y, Stokes IAF, Poulin F, Gardner-Morse M, Labelle $\mathrm{H}$ (2003) Biomechanical modeling of posterior instrumentation of the scoliotic spine. Comput Methods Biomech Biomed Eng 6(1):27-32. ISSN 1025-5842

Bathe KJ (1996) Finite element procedures. Prentice-Hall, Englewood Cliffs, NJ

Bisshopp KE, Drucker DC (1945) Large deflection of cantilever beams. Q Appl Math 3(3): 272-275. ISSN 0033569X

Bridwell KH (1999) Surgical treatment of idiopathic adolescent scoliosis. Spine 24(24):2607-2616

Cheh G, Lenke LG, Lehman RA, Kim YJ, Nunley R, Bridwell KH (2007) The reliability of preoperative supine radiographs to predict the amount of curve flexibility in adolescent idiopathic scoliosis. Spine 32(24):2668-2672

Cheung KMC, Luk KDK (2011) Prediction of correction of scoliosis with use of the fulcrum bending prediction of correction of scoliosis with use of the fulcrum bending radiograph. J Bone Jt Surg 79:1144-1150
Cobb JR (1948) Outline of the study of scoliosis. AAOS Instructional Lectures. Struct Multidisc Optim 5(5):261-75

Dumas R, Lafage V, Lafon Y, Steib J-P, Mitton D, Skalli W (2005) Finite element simulation of spinal deformities correction by in situ contouring technique. Comput Methods Biomech Biomed Eng 8(5):331-337

Elias de Oliveira M, Hasler CC, Studer D, Schneider J, Büchler P (2011) A software tool for assessment of the spinal flexibility. In: Proceedings of the XXIII congress of the international society of biomechanics. Brussels, Belgium,

Gardner-Morse M, Stokes IA (1994) Three-dimensional simulations of the scoliosis derotation maneuver with Cotrel-Dubousset instrumentation. J Biomech 27(2):177-181

Gardner-Morse MG, Laible JP, Stokes IA (1990) Incorporation of spinal flexibility measurements into finite element analysis. J Biomech Eng 112(4):481-483

Ghista DN, Viviani GR, Subbaraj K, Lozada PJ, Srinivasan TM, Barnes G (1988) Biomechanical basis of optimal scoliosis surgical correction. J Biomech 21(2):77-88. ISSN 0021-9290

Hartley R, Zisserman A (2000) Multiple view geometry, vol 6. Cambridge University Press

Kennedy J, Eberhart R (1995) Particle swarm optimization. Opt Express 4:1942-1948. ISSN 10944087

King HA, Moe JH, Bradford DS, Winter RB (2011) The selection of fusion levels in thoracic idiopathic scoliosis selection of fusion idiopathic levels scoliosis in thoracic. Surgery 65:13021313

Klepps SJ, Lenke LG, Bridwell KH, Bassett GS, Whorton J (2001) Prospective comparison of flexibility radiographs in adolescent idiopathic scoliosis. Spine 26(5):E74-E79

Krismer M, Bauer R, Sterzinger W (1992) Scoliosis correction by Cotrel-Dubousset instrumentation. The effect of derotation and three dimensional correction. Spine 17(8 Suppl):S263-S269

Leborgne P, Skalli W, Lecire C, Dubousset J, Zeller R, Lavaste F (1999) Simulations of CD surgery on a personalized finite element model: preliminary results. In: Proceedings of the IRSSD, 28th June-1st July 1999

Lenke LG, Betz RR, Bridwell KH, Clements DH, Harms J, Lowe TG, Shufflebarger HL (1998a) Intraobserver and interobserver reliability of the classification of thoracic adolescent idiopathic scoliosis. J Bone Jt Surg 80(8):1097-1106

Lenke LG, Betz RR, Bridwell KH, Clements DH, Harms J, Lowe TG, Shufflebarger HL (1998b) Intraobserver and interobserver reliability of the classification of thoracic adolescent idiopathic scoliosis. J Bone Jt Surg 80(8):1097-1106

Lenke LG, Betz RR, Harms J, Bridwell KH, Clements DH, Lowe TG, Blanke K (2001) Adolescent idiopathic scoliosis: a new classification to determine extent of spinal arthrodesis. J Bone Jt Surg, Am Vol 83-A(8):1169-81. ISSN 0021-9355

Luk KD, Cheung KM, Lu DS, Leong JC (1998) Assessment of scoliosis correction in relation to flexibility using the fulcrum bending correction index. Spine 23(21):2303-2307

Ogon M, Giesinger K, Behensky H, Wimmer C, Nogler M, Bach CM, Krismer M (2002) Interobserver and intraobserver reliability of Lenke's new scoliosis classification system. Spine 27(8): 858-862

Panjabi MM, Brand RA, White AA (1976) Three-dimensional flexibility and stiffness properties of the human thoracic spine. J Biomech 9(4): 185-192

Petit Y, Aubin CE, Labelle H (2004) Patient-specific mechanical properties of a flexible multi-body model of the scoliotic spine. Med Biol Eng Comput 42(1):55-60

Polly DW, Sturm PF (1998) Traction versus supine side bending. Which technique best determines curve flexibility? Spine 23(7):804-808 
Poulin F, Aubin CE, Stokes IA, Gardner-Morse M, Labelle H (1998) Biomechanical modeling of instrumentation for the scoliotic spine using flexible elements: a feasibility study. Ann Chir 52(8): 761-767

Powell MJD (1964) An efficient method for finding the minimum of a function of several variables without calculating derivatives. Comput J 7(2):155-162. doi:10.1093/comjnl/7.2.155

Puno RM, An K-C, Puno RL, Jacob A, Chung S-S (2003) Treatment recommendations for idiopathic scoliosis: an assessment of the Lenke classification. Spine 28(18):2102-2114; discussion 21142115

Robitaille M, Aubin CE, Labelle H (2007) Intra and interobserver variability of preoperative planning for surgical instrumentation in adolescent idiopathic scoliosis. European spine journal official publication of the European Spine Society the European Spinal Deformity Society and the European Section of the Cervical Spine Research Society. Struct Multidisc Optim 16(10):16041614

Rosenbrock HH (1960) An automatic method for finding the greatest or least value of a function. Comput J 3(3):175-184. doi:10.1093/comjnl/3.3.175. ISSN 00104620
Shi Y, Eberhart R (1998) A modified particle swarm optimizer. Appl Math Comput 189(2):69-73. ISSN 15499596

Stokes IAF, Gardner-Morse M (1999) Simulation of surgical maneuvers with C-D instrumentation. IOS Press, Amsterdam, pp 377380

Stokes IAF, Gardner-Morse M, Aubin CE, Labelle H (1999) Biomechanical simulations for planning of scoliosis surgery, threedimensional analysis of spinal deformities, pp 343-346

Vanderby R, Daniele M, Patwardhan A, Bunch W (1986) A method for the identification of in-vivo segmental stiffness properties of the spine. J Biomech Eng 108(4):312-316

Vaughan JJ, Winter RB, Lonstein JE (1996) Comparison of the use of supine bending and traction radiographs in the selection of the fusion area in adolescent idiopathic scoliosis. Spine 21(21):24692473

Vedantam R, Lenke LG, Bridwell KH, Linville DL (2000) Comparison of push-prone and lateral-bending radiographs for predicting postoperative coronal alignment in thoracolumbar and lumbar scoliotic curves. Spine 25(1):76-81

Zienkiewicz OC, Taylor RL, Zhu JZ (2005) The finite element method: its basis and fundamentals. Butterworth-Heinemann 